\title{
Influence of the Condition with and without External Support on the Strength of Hip Flexor in Supine in Subjects without Core Stabilization
}

\author{
In-Cheol Jeon \\ Department of Physical Therapy, College of Life and Health Science, Hoseo University, Asan, Republic of Korea
}

\begin{abstract}
Purpose: This study was performed to investigate the influence of the condition with and without external support on the strength of hip flexor in supine position in subjects without core stabilization. Hip flexor muscles are very functional in the hip joint structures. Therefore, it is essential to evaluate the strength of hip flexor in a clinical and precise way.

Methods: Twenty subjects participated in this study. The double bent leg-lower test was used to evaluate subjects without core stabilization. The strength of hip flexor muscles was evaluated in supine position, both with and without external support condition. The paired t-test was used to compare the strength of hip flexor muscles according to external support. The level of statistical significance was at $\alpha=0.05$. The intra-rater reliability of the repeated measures of hip flexor strength was estimated by calculating the intra-class correlation coefficients (ICC).

Results: In subjects without core stabilization, the strength of hip flexor in supine was greater with external support than that without external support $(p<0.05)$. In addition, the intra-rater reliability with an $\operatorname{ICC}(3,1)$ of the strength measurement of hip flexor with external support was higher than that without external support.

Conclusion: In subjects without core stabilization, the condition with external support can contribute to the strength of hip flexor in supine position and the strength measurement of hip flexor should be considered with the condition with and without external support.
\end{abstract}

Keywords: Core stabilization, Hip joint, Rehabilitation

\section{INTRODUCTION}

Core stabilization is defined as an internal stabilization made by the isometric contraction of the lumbar and abdominal muscles to maintain stability. It has been also known as core strengthening and dynamic stabilization. ${ }^{2}$ Core stabilization has been recommended to manage chronic lower back pain, ${ }^{3}$ improve the balance, ${ }^{4}$ and prevent musculoskeletal injuries. ${ }^{5,6}$ Weakness and poor endurance of hip flexor muscles have been reported in subjects with lower extremity injuries and low back pain., ${ }^{5,7}$ The previous findings also reported that subjects with patellofemoral joint pain have weaker hip flexors and external rotators than the control group. ${ }^{89}$

Because hip flexors have a role of pelvic stabilizers during active straight leg raising (ASLR), they are considered as the essential muscles surrounding the hip joint structures. ${ }^{10}$ Insufficient hip flexor muscles may result in

Received Nov 10, 2020 Revised Dec 13, 2020

Accepted Dec 21, 2020

Corresponding author In-Cheol Jeon

E-mail jeon6984@hoseo.edu an uncontrolled movement in lumbopelvic region ${ }^{11}$ or an anterior gliding syndrome in hip joint. ${ }^{3}$ Accurate assessment of these compensatory mechanisms can be necessary to interpret and evaluate hip flexor function objectively. ${ }^{12}$ Before and after total joint arthroplasty, the individuals were affected by hip flexor muscle deficits, such as weakness ${ }^{13}$ and functional limitations. ${ }^{14}$

Based on previous studies, the core stabilization has been generally assessed with subjects in supine $e^{15,16}$ and side-lying positions. ${ }^{4,17}$ Comerford and Mottram ${ }^{11}$ reported that the double bent leg lower test can be used to evaluate the core stabilization in a crook-lying position. This test is performed with a pressure biofeedback unit (PBU) positioned in the lumbopelvic region. The subject could maintain the pressure of a $\mathrm{PBU}$ at $40 \pm 10$ $\mathrm{mmHg}$ during both bent leg lowering. However, in subjects without core stabilization, the value of the pressure was changed above $10 \mathrm{mmHg}$. The

Copylight (C)2020 The Korean Society of Physical Therapy

This is an Open Access article distribute under the terms of the Creative Commons Attribution Non-commercial License (https:// creativecommons.org/license/by-nc/4.o.) which permits unrestricted non-commercial use, distribution, and reproduction in any medium, provided the original work is properly cited. 
previous study, the pelvic rotation control method as an internal stabilization and the pelvic compression belt as an external stabilization were compared to investigate the lumbopelvic stability during ASLR. However, lack of study on hip flexor strength in supine position was in subjects without core stabilization. This study investigates the influence of the condition with and without external support on hip flexor muscle strength in supine position in subjects without core stabilization. Hip flexion in supine position is the conventional movement for testing strength of various hip flexors including iliopsoas muscle. Investigating the role of the condition with external support for core stabilization in supine position will be clinically useful for prescription of the effective hip flexor strengthening exercise and precise strength measurement.

Therefore, we hypothesized that the strength of hip flexor in supine position with external support would be significantly greater than that without external support in subjects without core stabilization. Moreover, the intra-rater reliability would be higher with external support than that without external support in subjects without core stabilization. The results of this study would guide the prefer treatment regarding the clinical techniques for testing hip flexor strength.

\section{METHODS}

\section{Subjects}

In total, thirty-eight subjects were assessed for eligibility. Eighteen subjects were excluded because of the test criteria. Twenty subjects without core stabilization were recruited for this study. The exclusion criteria were disorders with neuromuscular or musculoskeletal aspects, and pain in any parts of the body during tasks (Table 1). The experimental protocols were explained in detail to all subjects and they all provided written informed consent in keeping with the ethical principles of the Declaration of Helsinki to participate this study. This study was approved by the relevant Institutional Review Board.

\section{Experimental methods}

The unilateral maximal isometric strength of hip flexor muscles was quantified in supine position to assess the strength of hip flexor using the dominant leg, as the leg preferred for kicking a ball. ${ }^{18,19}$ The strength measurement for the dominant side was performed as a tested hip side both with and without external support in subjects without core stabilization as a cross sectional study design. For the test-retest reliability of the hip flexor strength measurement, two test sessions with resting time were performed
Table 1. General characteristics of the subjects.

$(N=20)$

\begin{tabular}{lc}
\hline & Mean \pm SD \\
\hline Age $(\mathrm{yr})$ & $23.2 \pm 3.1$ \\
Height $(\mathrm{cm})$ & $169.8 \pm 6.8$ \\
Weight $(\mathrm{kg})$ & $70.7 \pm 9.6$ \\
Gender $(\mathrm{M} / \mathrm{F})$ & $12 / 8$
\end{tabular}

SD: Standard deviation.

at the same time.

\section{1) Measurement \\ (1) Testing for Core Stabilization}

The double bent leg lower test was used, as recommended by Comerford and Mottram ${ }^{11}$, to evaluate subjects without core stabilization. A PBU was positioned on the lumbopelvic region. The subjects were asked to get in a crook-lying position. The PBU pressure was adjusted to $40 \mathrm{mmHg}$. The subjects were asked to maintain the PBU at $40 \pm 10 \mathrm{mmHg}$, without holding their breath, while their legs were moving. The subjects were also asked to hold the PBU at $40 \mathrm{mmHg}$ with the hip joint flexed at $90^{\circ}$ for 5 seconds and with the heels held just above the table for 5 seconds. Specifically, the subjects were asked to dissociate movement through the benchmark range of bilateral independent hip extension from a $90^{\circ}$ to a $45^{\circ}$ hip flexion while maintaining the PBU pressure. The subjects without core stabilization was defined as in a pressure difference of the lumbopelvic region that exceeded or decreased above $10 \mathrm{mmHg}$ during the double bent leg lower test (Figure 1).11

\section{(2) The strength measurement of hip flexor muscles}

The isometric maximal voluntary contraction the strength of hip flexor muscles was measured with a tensiometer (Smart KEMA pulling sensor, Factorial Holdings Co., Ltd., Seoul, Korea). The strap location of the pulling sensor was positioned at $5 \mathrm{~cm}$ proximal to the patella and marked with a straight line at the same region on the skin to minimize the regional difference. ${ }^{20}$ The length of the strap was adjusted to each hip angle reaching $90^{\circ}$ in supine position. After these procedures, the same task was re-measured using the same procedure after 2 minutes of rest. In order to carry out the experimental procedure, the examiners were familiarized with the pulling sensor before performing the actual measurements. The tensiometer consists of force-detecting system with measuring forces of up to 100 $\mathrm{kg}, 0.1 \mathrm{~kg}$ accuracy, and $0.1 \mathrm{~kg}$ resolution. Before any measurements were taken, the subjects performed a warm-up of submaximal-speed jogging for 5 minutes to prevent discomfort or pain during the exercises. ${ }^{21}$ 

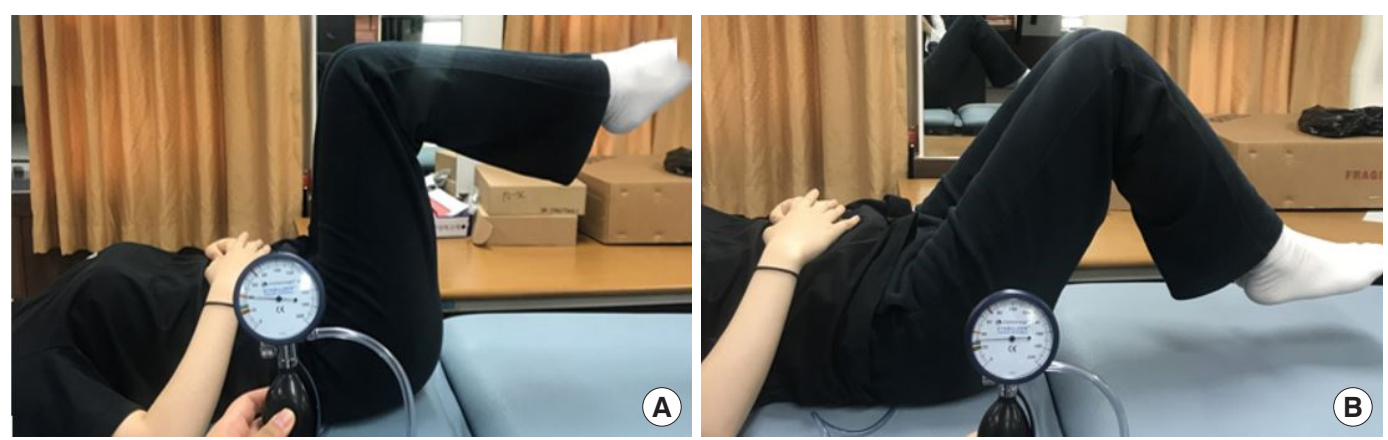

Figure 1. Testing for Core Stabilization. (A) Initial position, (B) Final position.
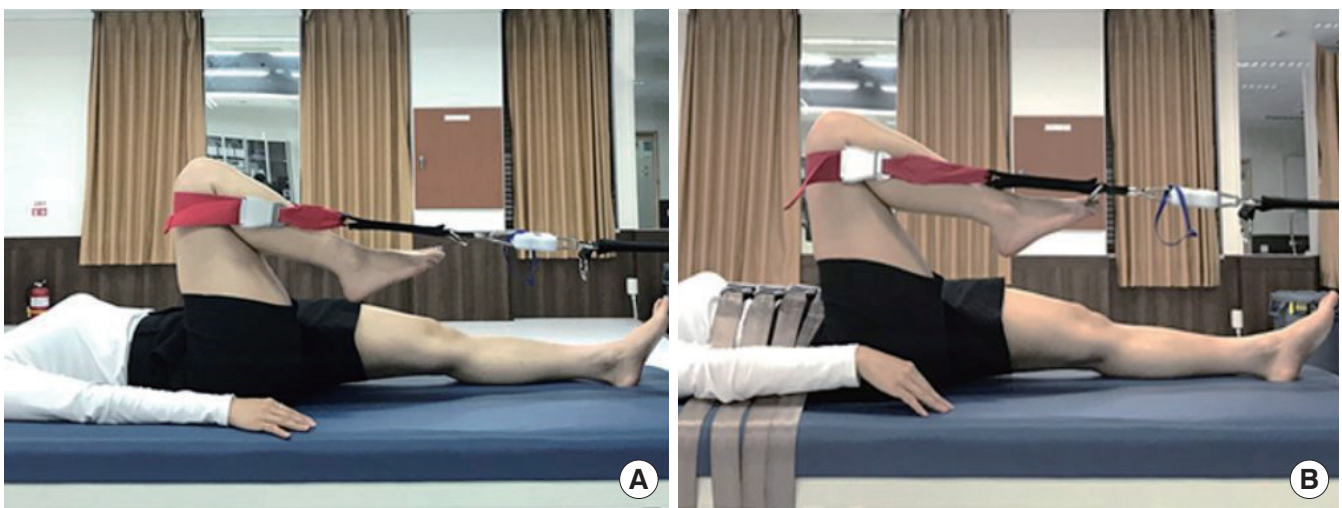

Figure 2. The strength measurement of hip flexor muscles. (A) Hip flexion without external support, (B) Hip flexion with external support.

The subjects without core stabilization were familiarized with the $90^{\circ}$ hip flexion in supine position, the examiner supervised each subjects during all exercises. Then, the isometric maximal voluntary contraction strength measurement was performed by the dominant side in supine position, both with and without external support. In addition, each test session was randomized to minimize the testing bias. In addition, the examiner was blinded to the strength measurement and any information of the subjects.

The subjects were in supine position on the table. The subjects were asked to perform hip flexion at $90^{\circ}$; for the same task with external support condition, non-elastic belt was applied to the subjects' abdomen to provide core stabilization in lumbopelvic region. During the measurements, the knee of tested limbs performed $90^{\circ}$ of hip flexion and the knee of non-tested limbs was straight during the isometric maximal voluntary contraction. ${ }^{20}$ The duration of these contractions was approximately 5 seconds. The measurements were performed three times with 2 minutes of resting time between trials to prevent fatigue. The maximal strength provided by the tensiometer (in kilograms) were retained (Figure 2).

\section{Statistical analysis}

The normal distribution of the measurement data was ensured with the one-sample Kolmogorov-Smirnov test. In subjects without core stabilization, the strength of hip flexor with and without external support was compared using the paired t-test. The level of statistical significance was set at $\alpha=0.05$. Intra-class correlation coefficients (ICCs) $(3,1)$ was used for intra-rater reliabilities within the same session to determine the intra-rater reliability of the strength measurements of hip flexor. The Statistical Package for the Social Sciences for Windows, ver. 18.0 (SPSS, Chicago, IL, USA) was used for statistical analysis. The ICC $(3,1)$ model was used to estimate intra-rater reliability of strength of hip flexor.

\section{RESULTS}

The strength of hip flexor in supine position with external support was significantly greater than that without external support $(25.16 \pm 6.13 \mathrm{~kg}$ without external support, $29.89 \pm 7.48 \mathrm{~kg}$ with external support; $\mathrm{t}=-15.11$, $\mathrm{p}<0.05$ )(Table 2, Figure 3).

The intra-rater reliability with an ICC $(3,1)$ of the hip flexor strength measurement with external support of 0.98 (95\% CI, 0.95-0.99, SEM, 0.56 
Table 2. The strength of hip flexor in subjects without core stabilization

\begin{tabular}{|c|c|c|c|c|}
\hline \multirow{2}{*}{ Groups } & \multicolumn{3}{|c|}{ Mean \pm standard deviation } & \multirow{2}{*}{$\mathrm{p}$ value } \\
\hline & Without External support & With External support & Changing values of strength $(\mathrm{kg})$ & \\
\hline Without core stabilization & $25.16 \pm 6.13$ & $29.89 \pm 7.48$ & $4.73 \pm 1.35$ & $<0.05$ \\
\hline
\end{tabular}

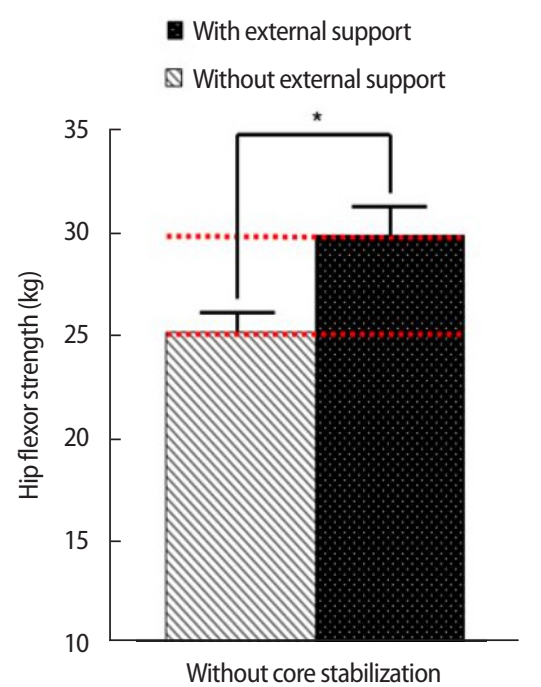

Figure 3. The difference in hip flexor strength in subjects without core stabilization according to the condition with vs. without external support. ${ }^{*} p<0.05$.

$\mathrm{kg}$ ) was higher than that without external support of 0.68 (95\% CI, $0.65-$ 0.79$, SEM, $0.96 \mathrm{~kg})$.

\section{DISCUSSION}

The muscle strength of hip flexor is important for individuals with weakness in their hip flexor muscles, especially for the rehabilitation of chronic lower back pain and improving hip joint stability. ${ }^{5,7,15}$ However, most of the clinical strength measurement and strengthening exercises of hip flexors are not commonly used with consideration of the core stabilization during a rehabilitation program. Moreover, a lack of consideration in the core stabilization may result in inappropriate training for the target muscles in individuals with insufficient core stabilization. ${ }^{3}$ In this study, the external support by using non-elastic belt was used to externally provide the core stabilization. The purpose of this study was to investigate the influence of the condition with and without external support on the strength of hip flexor muscle in supine position.

In this study, the strength of hip flexor was significantly greater with external support than that without external support in subjects without core stabilization (18.80\% strength difference in kg unit). Greater strength of hip flexor with external support was found, which may imply that the condition with external support during hip flexion acts to improve core stabilization and strength of hip flexor in supine position. The intra-rater reliability with an $\operatorname{ICC}(3,1)$ of the hip flexor strength measurement was higher in subjects with external support than that without external support.

There are possible reasons that may explain why external support for core stabilization contributed to the strength improvement of hip flexor in supine position. This result can be explained by the biomechanical elements. A previous study showed that proximal stability during lower extremity exercise can contribute to the facilitation of the iliopsoas and gluteus medius and maximus muscle attached to the spine and the pelvis. ${ }^{22,23}$ In addition, in a study by Jeon et al. ${ }^{24}$ also showed that the thickness of the iliopsoas was greater during ASLR in subjects without uncontrolled lumbopelvic rotation compare to subjects with uncontrolled lumbopelvic rotation. ${ }^{24}$ The core stabilization provided by the various stabilizing muscles such as transverse abdominal, internal oblique and iliopsoas could contribute to the strength of the lower extremities especially hip flexion. ${ }^{15} \mathrm{Al}-$ though the findings of the previous study could not be directly compared with this study because the stabilizing muscle activation as an internal stabilization and non-elastic belt as an external stabilization were different, the finding of this study was similar with the previous study indicating that internal and external stabilization can affected the strength of hip flexor muscle. ${ }^{15,24}$ In the previous study, the task was performed in supine position to investigate the effect of the iliopsoas as a stabilization during ASLR, hip flexor training with lumbar stabilization technique during ASLR can be recommended as an effective method of activating hip flexor muscles. ${ }^{24}$ In this study, the external support in abdominal region may act to provide proximal stability as a core stabilization contributing to greater strength of hip flexor compared to that without external support.

Hip flexors are important muscles in the hip joint structures because they have a role of hip joint stabilizers, during ASLR. ${ }^{10,15}$ In supine position, the core stabilization in abdominal region can provide counter-balanced force against load transfer through lower extremities. In addition, hip flexor can act to counter balance both pelvis and femur head against the resistance. ${ }^{3,11}$ However, in subjects without core stabilization, the ante- 
rior tilting of the pelvis and increased lumbar lordosis during hip flexion in supine position without external support resulted in a lengthened position of hip flexor and abdominal stabilizing muscles, causing difficulty in co-contraction of these muscles. To tie up with non-elastic band as an external support may provide external stability against resistance during hip flexion in supine position. The external support may minimize the anterior tilting of the pelvis and lumbar lordosis against the resistance direction during hip flexion in supine position, thus contributing to optimizing the length-tension of hip flexor muscle on the tested side. In addition, this condition with external support may contribute to higher intra-rater reliability compared to that without external support because the lumbopelvic compensations during the strength measurement might be minimized with external support in subjects without core stabilization.

External support for core stabilization can be recommended to improve hip flexor strength especially in subjects without core stabilization. The measurement of hip flexor strength in supine position should be performed separately in the condition with and without external supports in subjects without core stabilization.

This study had several limitations. First, surface EMG was not employed to investigate muscle activation during hip flexion in supine position because of external support condition used in this study. Therefore, further study is needed to investigate the muscle contractions by surface EMG of lumbopelvic muscles affecting core stabilization during hip flexion in supine position. Second, healthy subjects were not recruited as a control group. The findings in this study cannot be generalized to healthy subjects. Further study is needed to compare differences between individuals with and without core stabilization. Finally, this study was performed as a cross sectional study. Therefore, the abdominal drawing-in maneuver could not be used to activate abdominal stabilizing muscle in subjects without core stabilization. The further study is needed to determine whether longitudinal core stabilization training can improve hip flexor strength in supine position.

\section{ACKNOWLEDGEMENTS}

This research was supported by the Academic Research fund of Hoseo University in 2018 (No. 20180113).

\section{REFERENCES}

1. Kisner C, Colby LA, Borstad J. Therapeutic exercise: Foundations and techniques. Philadelphia, Fa Davis, 2017:451-75.

2. Akuthota V, Nadler SF. Core strengthening. Arch Phys Med Rehabil. 2004;85:86-92.

3. Sahrmann SA. Diagnosis and treatment of movement impairment syndromes. Missouri, Mosby, 2002:77-87.

4. Ekstrom RA, Donatelli RA, Carp KC. Electromyographic analysis of core trunk, hip, and thigh muscles during 9 rehabilitation exercises. J Orthop Sports Phys Ther. 2007;37(12):754-62.

5. Leetun DT, Ireland ML, Willson JD et al. Core stability measures as risk factors for lower extremity injury in athletes. Med Sci Sports Exerc. 2004;36(6):926-34.

6. Tyler TF, Nicholas SJ, Campbell RJ et al. The effectiveness of a preseason exercise program to prevent adductor muscle strains in professional ice hockey players. Am J Sports Med. 2002;30(5):680-3.

7. Leinonen V, Kankaanpää M, Airaksinen O et al. Back and hip extensor activities during trunk flexion/extension: effects of low back pain and rehabilitation. Arch Phys Med Rehabil. 2000;81(1):32-7.

8. Park KH, Ha SM, Kim SJ et al. Effects of the pelvic rotatory control method on abdominal muscle activity and the pelvic rotation during active straight leg raising. Man Ther. 2013;18(3):220-4.

9. Van Cant J, Pineux C, Pitance L et al. Hip muscle strength and endurance in females with patellofemoral pain: a systematic review with meta-analysis. Int J Sports Phys Ther. 2014;9(5):564-82.

10. Hu H, Meijer OG, Van Dieën JH et al. Is the psoas a hip flexor in the active straight leg raise? Eur Spine J. 2011;20(5):759-65.

11. Comerford M, Mottram S. Kinetic control: the management of uncontrolled movement. London, Churchill Livingstone, 2012:212-26.

12. Hardcastle P, Nade S. The significance of the Trendelenburg test. J Bone Joint Surg Br. 1985;67(5):741-6.

13. Shih CH, Du YK, Lin YH et al. Muscular recovery around the hip joint after total hip arthroplasty. Clin Orthop Relat Res. 1994;(302):115-20.

14. Perron M, Malouin F, Moffet $\mathrm{H}$ et al. Three-dimensional gait analysis in women with a total hip arthroplasty. Clin Biomech. 2000;15(7):504-15.

15. De Groot M, Pool-Goudzwaard AL, Spoor CW et al. The active straight leg raising test (ASLR) in pregnant women: differences in muscle activity and force between patients and healthy subjects. Man Ther. 2008;13 (1):68-74.

16. Jull G, Richardson C, Toppenberg R et al. Towards a measurement of active muscle control for lumbar stabilisation. Aust J Physiother. 1993; 39(3):187-93.

17. Cynn HS, Oh JS, Kwon OY et al. Effects of lumbar stabilization using a pressure biofeedback unit on muscle activity and lateral pelvic tilt during hip abduction in sidelying. Arch Phys Med Rehabil. 2006;87(11):1454-8.

18. Bolgla LA, Uhl TL. Electromyographic analysis of hip rehabilitation exercises in a group of healthy subjects. J Orthop Sports Phys Ther. 2005; 35(8):487-94.

19. Jung SH, Ha SM. The Effects of Lumbo-pelvic Stabilization on Hip Flexion Range of Motion Measurement. J Musculoskelet Sci Technol 2019; 3(2):49-53.

20. Jeon IC. Comparison of Test-retest Measurement Reliability of Iliopsoas Strength between Break and Make Test in Subjects with Lumbar Extension Syndrome. J Musculoskelet Sci Technol 2019; 3(2):54-8.

21. Hunter AM, St Clair Gibson A, Lambert MI et al. Effects of supramaximal exercise on the electromyographic signal. Br J Sports Med. 2003;37 


\section{TRPT $\begin{aligned} & \text { The Journal of } \\ & \text { Korean Physical Therapy }\end{aligned}$}

(4):296-9.

22. Kibler WB, Press J, Sciascia A. The role of core stability in athletic function. Sports Med. 2006;36(3):189-98.

23. Huxel Bliven KC, Anderson BE. Core stability training for injury prevention. Sports Health. F2013;5(6):514-22.
In-Cheol Jeon

24. Jeon IC, Kwon OY, Weon JH et al. Comparison of psoas major muscle thickness measured by sonography during active straight leg raising in subjects with and without uncontrolled lumbopelvic rotation. Man Ther. 2016;21:165-9. 